\title{
Fenologia e maturação da videira 'Cabernet Franc' em função de diferentes cargas de gemas em região de elevada altitude de Santa Catarina
}

\author{
Phenology and maturation of 'Cabernet Franc' grapevine due to different retained nodes \\ number in high altitude region of Santa Catarina State
}

\section{Douglas André Würz ${ }^{1 \star}$, Bruno Farias Bonin ${ }^{2}$, Alberto Fontanella Brighenti ${ }^{3}$, Adrielen Tamiris Canossa ${ }^{2}$, Juliana Reinehr ${ }^{2}$, Ricardo Allebrandt ${ }^{2}$, Betina Pereira de Bem², Leo Rufato ${ }^{2}$ e Aike Anneliese Kretzschmar ${ }^{2}$}

\author{
${ }^{1}$ Instituto Federal de Santa Catarina, Canoinhas-SC, Brasil *Autor para correspondência: douglaswurz@hotmail.com. \\ ${ }^{2}$ Universidade do Estado de Santa Catarina, Lages-SC, Brasil. \\ ${ }^{3}$ Empresa de Pesquisa Agropecuária e Extensão Rural de Santa Catarina, São Joaquim, SC, Brasil.
}

Submissão:17/12/2018 / Aceite: 01/07/2019

\begin{abstract}
RESUMO
Tem-se como objetivo desse trabalho avaliar o efeito do aumento da carga de gemas na fenologia e na maturação tecnológica e fenológica da videira Cabernet Franc em região de elevada altitude de Santa Catarina. O presente trabalho foi conduzido durante safra 2016/2017, em um vinhedo comercial, localizado no munícipio de São Joaquim. Os tratamentos consistiram em quatro diferentes níveis de cargas de gemas: 15, 30, 50 e 75 gemas planta- ${ }^{-1}$. Durante o ciclo vegetativo da videira foram avaliados os principais estádios fenológicos (brotação, floração, virada de cor e maturação para colheita), sendo avaliadas qualitativamente quanto qualitativamente. No momento da colheita foram coletadas 100 bagas por parcela para a determinação da maturação tecnológica e fenólica. O delineamento experimental utilizado foi o de blocos ao acaso, com quatro blocos e cinco plantas por parcela. Os dados foram submetidos à análise de variância (ANOVA) e comparados pelo Teste Tukey a $5 \%$ de probabilidade de erro. $\mathrm{O}$ aumento da carga de gemas resultou em maior duração da Brotação e Floração, enquanto para a duração do Verásion, não observou-se efeito das diferentes cargas de gemas. A maturação tecnológica não foi influenciada pelo aumento da carga de gemas, e nos intervalos de carga de gemas testadas (15 a 75 gemas/planta), obteve-se índices de maturação considerados adequados para a elaboração de vinhos de qualidade. $\mathrm{O}$ aumento da carga de gemas não proporcionou diferença significativa nos atributos físicoquímicos, na composição fenólica e coloração das bagas.
\end{abstract}

PALAVRAS-CHAVE: Vitis vinifera L., equilíbrio vegetativo, poda, maturação.

\begin{abstract}
The objective of this work is to evaluate the effect of load of buds on phenology and the technological and phenological maturation of Cabernet Franc grapevine in high-altitude region of Santa Catarina. The present work was conducted during the 2016/2017 harvest, in a commercial vineyard, located in the city of São Joaquim. The treatments consisted of four different levels of bud loads: 15, 30, 50, and 75 plant $^{-1}$ buds. During the vegetative cycle of the grapevine, the main phenological stages were evaluated (budding, flowering, color turning, and maturation for harvest), being evaluated qualitatively and qualitatively. At the time of harvest, 100 berries were collected per plot to determine technological and phenolic maturation. The experimental design was a randomized block design with four blocks and five plants per plot. The data were submitted to analysis of variance (ANOVA) and compared by Tukey test with a $5 \%$ probability of error. The increase of the bud load resulted in a longer duration of Budding and Flowering, whereas for the duration of Verásion, no effect of different bud loads was observed. Technological maturation was not influenced by the increase of the yolk load, and in the sample loading intervals ( 15 to 75 yolks/plant), maturation rates considered adequate for the elaboration of quality wines were obtained. The increase of the yolk load did not give a significant difference in the physical-chemical attributes, in the phenolic composition and color of the berries.
\end{abstract}

KEYWORDS: Vitis vinifera L., vegetative balance, pruning, ripening. 


\section{INTRODUÇÃO}

As regiões de elevada altitude de Santa Catarina, SC caracterizam-se por apresentar vinhedos entre 900 e 1400 metros acima do nível do mar, permitem fornecer matéria prima para elaboração de vinhos diferenciados por sua intensa coloração, aroma e acidez (MARCON FILHO et al. 2015, MALINOVSKI et al. 2016, WURZ et al. 2017a). No entanto, observa-se nessa região elevada disponibilidade hídrica (BEM et al. 2016), solos com altos teores de matéria orgânica (MAFRA et al. 2011), que resultam em crescimento vegetativo excessivo, baixo índice de fertilidade de gemas, podendo afetar a produtividade e a maturação das uvas (BRIGHENTI et al. 2014, WURZ et al. 2017b, WURZ et al. 2018).

Umas das alternativas para aumentar a produtividade dos vinhedos, e melhor o equilíbrio vegetativo é através da poda de inverno, aumentando a relação gemas planta ${ }^{-1}$. Alterar o número de gemas da videira durante a poda de inverno é a principal e mais barata forma de adequar o rendimento do vinhedo (WINKLER 1965). Contudo, ressalta-se que o aumento da carga de gemas/planta pode resultar em uma série de efeitos na videira, que vão desde a fenologia até qualidade química da uva.

Vários estudos relataram diferenças, em função da intensidade e tipo de poda, no vigor vegetativo e composição final das bagas de uvas tanto para consumo in natura (AHMAD et al. 2004) quanto para vinificação (KURTURAL et al. 2006, BINDON et al. 2008, O'DANIEL et al. 2012), além da fenologia da videira.

DIXON (2009) estudando a variedade Sauvignon Blanc, nas condições edafoclimáticas da Nova Zelândia, observou que plantas podadas com 24 gemas floresciam mais cedo que vinhas podadas com 48 gemas. Há algumas evidências que a poda com maior número de gemas pode atrasar a floração e diminuir a taxa de floração (DIXON 2009). Já em estudo realizado por GREVEN et al. (2015), também realizado Nova Zelândia, com a e mesma variedade, observou-se que o aumento da carga de gemas não apresentou efeito na duração de cada estádio fenológica da videira (brotação, floração e verásion).

A literatura fornece hoje evidências suficientes que a produtividade e a qualidade são descritas como uma curva ótima, onde a redução da qualidade dos frutos é observado com o aumento da produtividade (HOWELL 2001, HUNTER 1998, PONI et al. 2004). No entanto, vários estudos relacionam a poda mínima, ou maior carga de gemas/planta sugerem que a produtividade do vinhedo pode ser aumentada sem haver prejuízo da qualidade dos frutos (MARTINEZ DE TODA \& SANCHA 1999, INTRIERI et al. 2011, PONI et al. 2016). Trabalho realizado por GREVEN et al. (2015), o aumento de 24 para 72 gemas por planta resultou em aumento da produtividade de 4,8 para 12,7 ton/ha, e essa diferença na produtividade teve como consequência um atraso na época de florescimento e veráison, havendo portanto, um atraso no acúmulo de sólidos solúveis.

A busca pelo equilíbrio vegeto-produtivo é um desafio nas condições das regiões de elevada altitude de Santa Catarina, onde o clima úmido e solo fértil tornam o controle do crescimento um desafio, e nesse contexto tem-se como objetivo desse trabalho avaliar o efeito do aumento da carga de gemas na fenologia e na maturação tecnológica e fenológica da videira Cabernet Franc em região de elevada altitude de Santa Catarina.

\section{MATERIAL E MÉTODOS}

O presente trabalho foi conduzido durante safra 2016/2017, em um vinhedo comercial, localizado no

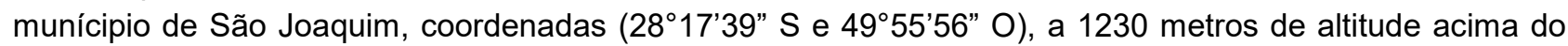
nível do mar. Utilizaram-se plantas de Cabernet Franc enxertadas sobre o porta-enxerto 'Paulsen 1103'. Os vinhedos foram implantados em 2004. O vinhedo caracteriza-se por apresentar plantas espaçadas de $3,0 \mathrm{x}$ $1,5 \mathrm{~m}$, em filas dispostas no sentido N-S, conduzidas em espaldeira, podadas em cordão esporonado duplo, a 1,2 m de altura e cobertas com tela de proteção anti-granizo, e com histórico de baixas produtividades.

Os solos da região enquadram-se nas classes Cambissolo Húmico, Neossolo Litólico e Nitossolo Háplico, desenvolvidos a partir de rocha riodacito e basalto (EMBRAPA 2004). O clima da região é classificado como 'Frio, Noites Frias e Úmido', Índice Heliotérmico de 1.714, precipitação pluvial média anual de $1.621 \mathrm{~mm}$ e a umidade relativa do ar média anual de 80\% (TONIETTO \& CARBONNAU 2004).

Os tratamentos consistiram em quatro diferentes níveis de cargas de gemas: 15, 30, 50 e 75 gemas planta $^{-1}$. A poda foi realizada para a Cabernet Franc no dia 08 de setembro de 2016. Deixou-se 8, 15, 25 esporões com duas gemas cada esporão para os tratamentos 15, 30 e 50 gemas por planta, e para o tratamento 75 gemas por planta, deixou-se 30 esporões com duas gemas, e duas varas com oito gemas cada, sendo portanto, este tratamento podada no sistema de poda mista, caracterizada pela presença de esporões e varas.

Durante o ciclo vegetativo da videira foram avaliados os principais estádios fenológicos (brotação, 
floração, virada de cor e maturação para colheita), sendo avaliadas qualitativamente quanto qualitativamente.

A avaliação da brotação ocorreu a cada três dias, avaliando-se o número de gemas em estádio de ponta verde por planta. Considerou-se $100 \%$ de brotação quando todas as gemas atingiram o estádio fenológico de ponta verde. A floração foi avaliada conforme metodologia proposta por GREVEN et al. (2015), onde 20 cachos por parcela foram demarcados, avaliando-se visualmente os cachos, atribuindo notas de 0,5,10, 25, 50, 75, 90, 95 e 100\% de florescimento de cada cacho. As avaliações foram feitas a cada três dias por um período de três semanas. Para determinar a virada de cor da variedade Cabernet Franc, foi atribuído uma escala de notas de 0, 5, 10, 25, 50, 75, 90, 95 e 100\%, considerando o número de bagas coloridas em relação ao total de bagas do cacho.

No momento da colheita foram coletadas 100 bagas por parcela, segundo metodologia proposta por RIZZON \& MIELLE (2002) para a determinação da maturação tecnológica e fenólica. As bagas foram levadas ao Laboratório de Enologia da UDESC de Lages, onde passaram pelo processo de pesagem e separação das cascas. A partir do mosto, obtido pela maceração da polpa, foram determinados os sólidos solúveis (ํㅏix), a acidez total titulável (meq $\mathrm{L}^{-1}$ ) e o $\mathrm{pH}$, conforme a metodologia proposta pelo Office International de la Vigne et du Vin (OIV 2009).

O teor de sólidos solúveis (SS) foi determinado utilizando um refratômetro digital para açúcar, marca Atago - Modelo B427286. A acidez total (AT) foi obtida através da titulação do mosto com solução alcalina padronizada de hidróxido de sódio $0,1 \mathrm{~mol} . \mathrm{L}^{-1}$, utilizando como indicador azul de bromotimol, sendo os resultados expressos em meq $\mathrm{L}^{-1}$. O potencial hidrogeniônico $(\mathrm{pH})$ foi registrado por meio de um potenciômetro de bancada marca lon - modelo Phb500, após calibração em soluções tampões conhecidas de $\mathrm{pH}$ 4,0 e 7,0.

As cascas separadas das amostras das bagas de Cabernet Franc passaram por um processo de extração para a obtenção do teor de compostos fenólicos e de cor da casca.

Para a obtenção das soluções extratos seguiu-se a metodologia descrita por MARCON FILHO et al. (2015). A concentração de polifenóis totais (PT) na casca foi determinada pelo método de espectrofotometria, descrito por SINGLETON \& ROSSI 1965, utilizando o reagente Folin-Ciocalteu (Vetec) e o ácido gálico como padrão, com leituras da absorbância em $760 \mathrm{~nm}$. O teor de antocianinas na casca foi determinado pelo método de espectrofotometria, descrito por RIZZON (2010). Método químico baseado na diferença de coloração das antocianinas em relação ao $\mathrm{pH}$, visto que a variação da intensidade corante em dois valores de $\mathrm{pH}$ é proporcional ao teor de antocianina.

A cor foi determinada pelo método de espectrofotometria, descrito por RIZZON (2010). O extrato foi diluído na proporção 1:10 e analisado em espectrofotômetro nos comprimentos de onda de $420 \mathrm{~nm}, 520 \mathrm{~nm}$ e $620 \mathrm{~nm}$. A cor foi mensurada pelos parâmetros de intensidade e tonalidade de cor, obtida através das fórmulas: Intensidade $=420+520+620 \mathrm{~nm}$ e Tonalidade $=420 / 520 \mathrm{~nm}$. As amostras foram analisadas em espectrofotômetro UV-VIS (Biospectro - Modelo SP220) e todas as análises foram realizadas em duplicata.

O delineamento experimental utilizado foi o de blocos ao acaso, com quatro blocos e cinco plantas por parcela. Os dados foram submetidos à análise de variância (ANOVA) e comparados pelo Teste Tukey a $5 \%$ de probabilidade de erro.

\section{RESULTADOS E DISCUSSÃO}

As variáveis fenológicas (\%brotação, \%floração e \%veráison) da variedade Cabernet Franc estão apresentadas na Tabela 1. Para a variável fenologia, avaliou-se o início (10\%) e o final $(90 \%)$ de cada fase fenológica da videira. Observou-se efeito da carga de gemas no tempo de duração da brotação e floração, enquanto para a veráison não observou-se efeito da carga de gemas. A data de início da brotação foi semelhante para ambos os tratamentos, variando de 16/set à 18/set. No entanto, houve diferenças entre a data final da fase de brotação da videira. As plantas podadas com 15 gemas, 30 gemas e 50 gemas atingiram $90 \%$ de gemas brotados em 29/set, 01/out e 05/out, respectivamente (Tabela 1). Já as plantas podadas com 75 gemas atingiram $90 \%$ de gemas brotadas apenas no dia 12/out, ou seja, uma duração de 25 dias entre o início e o final da brotação. As planas podadas com 15 gemas/planta e 30 gemas/planta apresentam uma duração de 13,7 e 14,7 dias, respectivamente.

Para as uvas viníferas, a brotação, floração, veráison e a colheita são os estádios fenológicos mais importantes no seu desenvolvimento (JONES \& DAVIS 2000). A duração entre esses estádios fenológicos varia muito em relação a cultivar, o clima e a localização do vinhedo. O manejo da videira, em particular a poda de inverno, pode influenciar a produtividade do vinhedo, consequentemente influenciar a sua fenologia, desde o desenvolvimento dos frutos até a colheita (JONES \& DAVIS 2000, PETRIE et al. 2000, 
GREVEN et al. 2015). O número de gemas por planta pode influenciar o tempo de brotação no ano subsequente, podendo ser utilizado até mesmo, como uma alternativa para evitar danos por geadas.

Tabela 1. Efeito da carga de gemas na fenologia (\% brotação, \% floração e \% veráison) da videira Cabernet Franc (Vitis vinífera L.) em região de elevada altitude de Santa Catarina. Safra 2017.

Table 1. Effect of bud load on phenology (\% bud break, \% flowering, and \% veráison) of Cabernet Franc grapevine (Vitis vinifera L.) in high-altitude region of Santa Catarina. 2017 Harvest.

\begin{tabular}{|c|c|c|c|c|c|}
\hline \multirow{2}{*}{ Brotação (\%) } & \multicolumn{4}{|c|}{ Carga de Gemas } & \multirow{2}{*}{$\frac{C V}{(\%)}$} \\
\hline & 15 & 30 & 50 & 75 & \\
\hline \multicolumn{6}{|l|}{2017} \\
\hline $10 \%$ & $17 /$ set & $18 /$ set & $16 /$ set & $16 /$ set & - \\
\hline $50 \%$ & $23 /$ set & $24 /$ set & $25 /$ set & $25 /$ set & - \\
\hline $90 \%$ & $29 /$ set & 01/out & 05/out & 12/out & - \\
\hline Duração (dias) & $13,7 \mathrm{c}$ & $14,7 \mathrm{c}$ & $18,5 \mathrm{~b}$ & $25,7 \mathrm{a}$ & 10,2 \\
\hline \multirow{2}{*}{ Floração (\%) } & \multicolumn{4}{|c|}{ Carga de Gemas } & $\mathrm{CV}$ \\
\hline & 15 & 30 & 50 & 75 & $(\%)$ \\
\hline \multicolumn{6}{|l|}{2017} \\
\hline $10 \%$ & $20 /$ nov & $20 /$ nov & $16 /$ nov & $17 /$ nov & - \\
\hline $50 \%$ & $24 /$ nov & $24 /$ nov & $22 /$ nov & $22 /$ nov & - \\
\hline $90 \%$ & $27 /$ nov & $27 /$ nov & $16 /$ nov & $26 /$ nov & - \\
\hline Duração (dias) & $7,7 \mathrm{~b}$ & $8,7 \mathrm{~b}$ & $10,2 \mathrm{ab}$ & $13 a$ & 11,5 \\
\hline \multirow{2}{*}{ Verásion (\%) } & \multicolumn{4}{|c|}{ Carga de Gemas } & CV \\
\hline & 15 & 30 & 50 & 75 & $(\%)$ \\
\hline \multicolumn{6}{|l|}{2017} \\
\hline $10 \%$ & $04 / \mathrm{fev}$ & $05 / \mathrm{fev}$ & $05 / \mathrm{fev}$ & $06 / \mathrm{fev}$ & - \\
\hline $50 \%$ & $09 / \mathrm{fev}$ & $10 /$ fev & $12 / \mathrm{fev}$ & $14 / \mathrm{fev}$ & - \\
\hline $90 \%$ & $14 / \mathrm{fev}$ & $17 / \mathrm{fev}$ & $18 / \mathrm{fev}$ & $20 / \mathrm{fev}$ & - \\
\hline Duração (dias) & 13,2 ns & 13,2 ns & 13,2 ns & 14,2 ns & 10,6 \\
\hline
\end{tabular}

*Médias seguidas da mesma letra, na linha, não diferem entre si pelo teste Tukey a 5\% de probabilidade de erro. ns = não significativo pela análise de variância (ANOVA) a $5 \%$ de probabilidade de erro.

Para o estádio fenológico floração, observou-se um menor tempo de duração para os tratamentos podados com 15 e 30 gemas/planta, apresentam uma duração de 7,7 e 8,7 dias. Assim como na brotação, as plantas podadas com 75 gemas apresentaram maior duração do período de floração, com 13 dias (Tabela 1). Dados observados por DIXON (2009), indicam que plantas de Sauvignon Blanc podadas com 24 gemas/planta florescem mais precocemente em relação a plantas podadas com 48 gemas/planta.

Diferentemente do observado nos estádios fenológicos brotação e floração, não observou-se efeito das diferentes cargas de gemas em relação ao tempo de duração da veráison (Tabela 1). $O$ início da veráison foi em 04/02, 05/02, 05/02 e 06/02 para as cargas de 15, 30, 50 e 75 gemas, respectivamente. Enquanto o final do veráison ocorreu em 14/02, 17/02, 18/02, 20/02. Em relação ao estádio fenológico veráison, TROUGHT \& BENNETT (2009) relatam que cachos provenientes de videiras com menores índices produtivos geralmente amadurecem antes daqueles de videiras de maiores produtividades. Contudo essa diferença não foi observada no presente trabalho, indicando que as diferentes cargas de gemas não afetaram a duração do período de verásion.

Trabalho realizado por GREVEN et al. (2015), ao avaliar as cargas de 24 a 72 gemas/planta, na variedade Sauvignon Blanc, não observou efeito do aumento da carga de gemas na duração dos estádios fenológicos avaliados (\% brotação, \% floração e \% verásion)

Apesar da influência da carga de gemas/planta nas videiras, o período dos estádios fenológicos observados nesse estudo assemelham-se aos observados por BORGHEZAN et al. (2011), nessa mesma região vitícola. Ressalta-se que o ciclo fenológico da videira em São Joaquim, SC, é mais tardio em comparação às demais regiões vitícolas brasileiras, devido as condições climáticas.

Existem algumas evidências que indicaram que uma maior carga de gemas/planta pode afetar a fenologia da planta, como atraso de brotação e floração (DIXON 2009). Esse atraso na ocorrência e duração dos diferentes estádios fenológicos pode afetar a curva de maturação dessas videiras, havendo a combinação de atributos maduros e não maduros nos frutos das videiras (PARR et al. 2005, PARR et al. 2007). No entanto não observou-se esse efeitos no presente trabalho, tendo em vista os valores referentes 
a maturação tecnológica das bagas da videira Cabernet Franc, descritos na Tabela 2. O conteúdo de sólidos solúveis e acidez total não foram influenciados pelas diferentes cargas de gemas/planta. $O$ conteúdo de sólidos solúveis variou de 21,3 a 21,7 ${ }^{\circ}$ Brix, enquanto a acidez total das bagas da videira variaram de 60,5 a 66,6 meq $\mathrm{L}^{-1}$.

Tabela 2. Efeito da carga de gemas na maturação tecnológica das bagas da videira Cabernet Franc (Vitis vinífera L.) em região de elevada altitude de Santa Catarina. Safra 2017.

Table 2. Effect of bud load on the technological maturation and staining of berries of the Cabernet Franc (Vitis vinifera L.) grapevine in high-altitude region of Santa Catarina. 2017 Harvest.

\begin{tabular}{|c|c|c|c|c|c|}
\hline & \multicolumn{4}{|c|}{ Carga de Gemas } & \multirow{2}{*}{$\frac{C V}{(\%)}$} \\
\hline & 15 & 30 & 50 & 75 & \\
\hline \multicolumn{6}{|l|}{2017} \\
\hline Sólidos Solúveis ( ${ }^{\circ}$ Brix) & $21,4^{\mathrm{ns}}$ & $21,3^{\text {ns }}$ & 21,7 ns & 21,7 ns & 1,3 \\
\hline Acidez Total (meq L-1) & 66,6 ns & $61,1^{\mathrm{ns}}$ & $60,5^{\mathrm{ns}}$ & 60,9 ns & 5,1 \\
\hline $\mathrm{pH}$ & $3,08 \mathrm{c}$ & $3,10 \mathrm{bc}$ & $3,15 a b$ & $3,16 \mathrm{a}$ & 0,8 \\
\hline
\end{tabular}

*Médias seguidas da mesma letra, na linha, não diferem entre si pelo teste Tukey a $5 \%$ de probabilidade de erro. ns = não significativo pela análise de variância (ANOVA) a 5\% de probabilidade de erro.

Para a maturação tecnológica, observou-se efeito da carga de gemas apenas para a variável pH. Os maiores valores de $\mathrm{pH}$ foram observados nas cargas de gemas de 50 e 75 gemas/planta, apresentando 3,15 e 3,16, respectivamente. Para a carga de 15 gemas/planta, observou-se o menor valor de $\mathrm{pH}$, com 3,08 .

Vários estudos relacionam a poda mínima, ou maior carga de gemas/planta (MARTINEZ DE TODA \& SANCHA 1999, INTRIERI et al. 2011, PONI et al. 2016), sugerem que a produtividade do vinhedo pode ser aumentada sem haver prejuízo da qualidade dos frutos.

Os valores obtidos neste trabalho podem ser considerados adequados a elaboração de vinhos tintos e estão de acordo com outros estudos realizados na Serra Gaúcha/Brasil (RIZZON \& MIELE 2002, MANDELLI et al. 2008), em Bordeaux/França (PEREIRA et al. 2005) e na Nova Zelândia (FRIEND \& TROUGHT 2007). Em geral, para a elaboração de vinhos de qualidade recomendam-se para o mosto, teores de sólidos solúveis acima de $20^{\circ}$ Brix, acidez total menor que 135 meq L-1 e pH menor que 3,5 (JACKSON 2014). Todos os índices citados acima foram observados nas diferentes cargas de gemas testados no presente trabalho.

O efeito da carga de gemas/planta na composição fenólica das bagas da videira Cabernet Franc estão descritas na tabela 3. Para todas as variáveis avaliadas (coloração Abs 420 nm, Abs 520 nm, Abs $620 \mathrm{~nm}$, intensidade de cor, tonalidade de cor, polifenóis totais e antocianinas) não observou-se diferença significativa da carga de gemas/planta.

Tabela 3. Efeito da carga de gemas na composição fenólica e coloração da película das bagas da videira Cabernet Franc (Vitis vinífera L.) em região de elevada altitude de Santa Catarina. Safra 2017.

Table 3. Effect of bud load on phenolic composition and film staining of berries of Cabernet Franc grapevine (Vitis vinifera L.) in high-altitude region of Santa Catarina. 2017 Harvest.

\begin{tabular}{lccccc}
\hline & \multicolumn{4}{c}{ Carga de Gemas } & CV \\
\cline { 2 - 5 } & 15 & 30 & 50 & 75 & $(\%)$ \\
\hline \multicolumn{1}{c}{ 2017 } & & & & \\
Coloração (Abs 420 nm) & $0,435^{\mathrm{ns}}$ & $0,479 \mathrm{~ns}$ & $0,455^{\mathrm{ns}}$ & $0,435^{\mathrm{ns}}$ & 6,7 \\
Coloração (Abs 520 nm) & $0,457^{\mathrm{ns}}$ & $0,482^{\mathrm{ns}}$ & $0,463^{\mathrm{ns}}$ & $0,458^{\mathrm{ns}}$ & 5,7 \\
Coloração (Abs 620 nm) & $0,169^{\mathrm{ns}}$ & $0,169 \mathrm{~ns}$ & $0,152^{\mathrm{ns}}$ & $0,148 \mathrm{~ns}$ & 12,4 \\
Intensidade de Cor & $1,061^{\mathrm{ns}}$ & $1,131^{\mathrm{ns}}$ & $1,071^{\mathrm{ns}}$ & $1,042^{\mathrm{ns}}$ & 3,8 \\
Tonalidade de Cor & $0,957^{\mathrm{ns}}$ & $0,997^{\mathrm{ns}}$ & $0,985^{\mathrm{ns}}$ & $0,947^{\mathrm{ns}}$ & 9,2 \\
Polifenóis Totais (mg L-1) & $1502,0^{\mathrm{ns}}$ & $1424,9^{\mathrm{ns}}$ & $1314,5^{\mathrm{ns}}$ & $1483,3^{\mathrm{ns}}$ & 8,4 \\
Antocianinas (mg L-1) & $202,5^{\mathrm{ns}}$ & $199,6^{\mathrm{ns}}$ & $203,8^{\mathrm{ns}}$ & $198,6^{\mathrm{ns}}$ & 10,1 \\
\hline
\end{tabular}

*Médias seguidas da mesma letra, na linha, não diferem entre si pelo teste Tukey a $5 \%$ de probabilidade de erro. ns = não significativo pela análise de variância (ANOVA) a 5\% de probabilidade de erro. 
Os compostos fenólicos apresentam correlação negativa com o vigor, devido à redução da síntese fenólica, de modo geral, baixos rendimentos são responsáveis pelo maior acúmulo de polifenóis totais (REYNOLDS et al. 2007, CANON et al. 2014), no entanto, não foi o que observou-se nesse estudo, visto que o aumento da carga de gemas planta-1 não apresentou efeito significativo no conteúdo de polifenóis totais das bagas, indicando a importância do equilíbrio vegeto-produtivo.

\section{CONCLUSÃO}

O aumento da carga de gemas resultou em maior duração da brotação e floração, enquanto para a duração do verásion, não observou-se efeito das diferentes cargas de gemas.

A maturação tecnológica não foi influenciada pelo aumento da carga de gemas, e nos intervalos de carga de gemas testadas (15 a 75 gemas/planta), obteve-se índices de maturação considerados adequados para a elaboração de vinhos de qualidade.

O aumento da carga de gemas não proporcionou diferença significativa nos atributos físico-químicos, na composição fenólica e coloração das bagas.

\section{REFERÊNCIAS}

AHMAD W et al. 2004. Effect of pruning severity on growth behavior of spur and bunch morphology of grapes (Vitis vinifera L.) cv. Perlette. International Journal of Agriculture and Biology 6: 160-161.

BEM BP et al. 2016. Effect of four training systems on the temporal dynamics of downy mildew in two grapevine cultivars in southern Brazil. Tropical Plant Pathology 41: 370-379.

BINDON K et al. 2008. Influence of partial root zone drying on the composition and accumulation of anthocyanins in grape berries (Vitis vinifera cv. Cabernet sauvignon). Australian Journal and Grape Wine Research 14: 91-103.

BORGHEZAN M et al. 2011. Comportamento vegetativo e produtivo da videira e composição da uva em São Joaquim, Santa Catarina. Pesquisa Agropecuária Brasileira 46: 398-405.

BRIGHENTI AF et al. 2014. Desempenho vitícola de variedades autóctones italianas em condição de elevada altitude no Sul do Brasil. Pesquisa Agropecuária Brasileira 49: 465-474.

CANON PM et al. 2014. Red Wine Phenolic: the effects of summer pruning and cluster thinning. Ciência e Investigacion Agraria 41: 235-248.

DIXON RA 2009. The influence of vine vigour and crop load on Sauvignon blanc vine growth and fruit composition in Marlborough, New Zealand. (Thesis). Canterbury: Lincoln University. 94p.

EMBRAPA 2004. Empresa Brasileira de Pesquisa Agropecuária. Solos do Estado de Santa Catarina. Rio de Janeiro: Embrapa Solos. 726p. (Boletim de pesquisa e desenvolvimento 46).

FRIEND AP \& TROUGHT MCT. 2007. Delayed winter spur-pruning in New Zealand can alter yield components of Merlot grapevines. Australian Journal of Grape and Wine Research 13: 157-164.

GREVEN MM et al. 2015. Influence of retained node number on Sauvignon Blanc grapevine phenology in a cool climate. Australian Journal of Grape and Wine Research 21: 209-301.

HOWELL GS 2001. Sustainable grape productivity and the growth-yield relationship: A review. American Journal of Enology and Viticulture 52: 165-174.

HUNTER JJ 1998. Plant spacing implications for grafted grapevine II. Soil water, plant water relations, canopy physiology, vegetative and reproductive characteristics, grape composition, wine quality and labour requirements. South African Journal Enololgy and Viticulture 19: 35-51.

INTRIERI CA 2011. Novel Mechanized Grapevine Training System. American Journal of Enology and Viticulture 62: $312-$ 318.

JACKSON RS 2014. Wine Science: principles and applications. 4.ed. Rio de Janeiro: Elsevier. 978p.

JONES GV \& DAVIS RE 2000. Climate influences on grapevine phenology, grape composition, and wine production and quality for Bordeaux, France. American Journal of Enology and Viticulture 51: 249-261.

KURTURAL SK et al. 2006. Effects of pruning and cluster thinning on yield and fruit composition of 'Chambourcin' grapevines. HortTechnology 16: 233-240.

MAFRA SHM et al. 2011. Atributos químicos do solo e estado nutricional de videira Cabernet Sauvignon (Vitis vinifera L.) na Serra Catarinense. Revista de Ciências Agroveterinárias 10: 44-53.

MALINOVSKI LI et al. 2016. Viticultural performance of Italian grapevines in high altitude regions of Santa Catarina State, Brazil. Acta Horticulturae 1115: 203-210.

MANDELLI F et al. 2008. Efeito da Poda Verde na composição físico-química do mosto da uva Merlot. Revista Brasileira de Fruticultura 30: 667-674.

MARCON FILHO JL et al. 2015. Raleio de cachos sobre o potencial enológico da uva 'Cabernet Franc' em duas safras. Ciencia Rural 45: 2150-2156.

MARTINEZ DE TODA F \& SANCHA JC 1999. Long-term effects of simulated mechanical pruning on Grenache vines under drought conditions. American Journal of Enology and Viticulture 50: 87-90.

O'DANIEL SB et al. 2012. Effects of balanced pruning severity on Traminette (Vitis spp.) in a warm climate. American Journal of Enology and Viticulture 63: 284-290.

OIV 2009. Recueil des méthodes internationales d'analyse des vins et des moûts. Paris: Office international de la vigne 
et du vin. $368 \mathrm{p.}$

PARR WV et al. 2005. Aroma and flavour of New Zealand Sauvignon Blanc. The Australian \& New Zealand Grapegrower \& Winemaker 100: 105-108.

PARR WV et al. 2007. The distinctive flavour of New Zealand Sauvignon blanc: Sensory characterisation by wine professionals. Food Quality and Preference 18: 849-861.

PEREIRA GE et al. 2005. 1H NMR and chemometrics to characterize mature grape berries in four wine-growing areas in Bordeaux, France. Journal of Agricultural and Food Chemistry 53: 6382-6389.

PETRIE PR et al. 2000. Influence of leaf ageing, leaf area and crop load on photosynthesis, stomatal conductance and senescence of grapevine (Vitis vinifera L. cv. Pinot noir) leaves. Vitis 39: 31-36.

PONI S et al. 2004. Performance of Croatina to short mechanical hedging: a successful case of adaptation. American Journal of Enology and Viticulture 55: 379-388.

PONI S et al. 2016. Mechanical winter pruning of grapevine: physiological bases and applications. Scientia Horticulturae 204: 88-98.

REYNOLDS AG et al. 2007. Magnitude of viticultural and enological effects. II. Relative impacts of cluster thinning and yeast strain on composition and sensory attributes of Chardonnay Musqué. American Journal of Enology and Viticulture 58: 25-41.

RIZZON LA 2010. Metodologia para análise de vinho. Brasília: Embrapa Informação Tecnológica. 120 p.

RIZZON LA \& MIELE A 2002. Acidez na vinificação em tinto das uvas Isabel, Cabernet Sauvignon e Cabernet Franc. Ciência Rural 32: 511-516.

SINGLETON VL \& ROSSI JA 1965. Colorimetry of total phenolics with phosphomolybdic - phosphotunestic acids reagents. American Journal of Enology and Viticulture 16: 144-158.

TONIETTO J \& CARBONNEAU A 2004. A multicriteria climatic classification system for grape-growing regions worlwide. Agricultural and Forest Meteorology 124: 81-97.

TROUGHT M \& BENNETT J 2009. Influence of training system on Sauvignon blanc grapevine performance 2004-2009. Auckland: Report to New Zealand Winegrowers.

WINKLER AJ 1965. Viticultura. México: Continental. 792p.

WURZ DA et al. 2017a. New wine-growing regions of Brazil and their importance in the evolution of Brazilian wine. BIO Web of Conferences 9: 1-4.

WURZ DA et al. 2017b. Agronomic performance of 'Cabernet Sauvignon' with leaf removal management in a highaltitude region of Southern Brazil. Pesquisa Agropecuária Brasileira 52: 869-876.

WURZ DA et al. 2018. Época de desfolha e sua influência no desempenho vitícola da uva 'Sauvignon Blanc' em região de elevada altitude. Revista de Ciências Agroveterinárias 17: 91-99. 